\title{
RELAC̣ÕES TRÓFICAS ENTRE AS CINCO ESPÉCIES DE PEIXES MAIS REPRESENTATIVAS NAS MARGENS DA LAGUNA DE JACAREPAGUÁ, RIO DE JANEIRO ${ }^{1}$
}

\author{
Luzia A. F. de Moraes ${ }^{2}$ \\ José V. Andreata ${ }^{3}$
}

\begin{abstract}
TROPHIC RELATIONS AMONG THE FIVE MOST REPRESENTATIVE FISH SPECIES IN THE JACAREPAGUÁ LAGOON EDGES, RIO DE JANEIRO. This study gives information on the feeding habits of 1,015 specimens, belonging to the five most abundant fish species in the Jacarepagua Lagoon, captured between March 1990 and February 1991, using trawl net. A feeding index was introduced to compare the relative importance of various food items, according to species standard length. A clustering analysis was calculated by using numerical coefficient of food items and defined two main groups: The Ist represented by Xenomelaniris brasiliensis (Quoy \& Gaimard, 1824) and Jenynsia lineata (Jenyns, 1842) considered omnivorous, fed mainly on insects, and the second including Tilapia rendalli (Boulenger, 1896), Phalloptychus januarius (Hensel, 1868) and Poecilia vivipara (Bloch \& Schneider, 1801), whose principal diet source was epibenthic cyanophytes, specially Microcystis sp. and Lyngbia sp.

KEY WORDS. Fishes, feeding habits, Jacarepagua Lagoon, Rio de Janeiro, Brazil
\end{abstract}

Abordou-se neste trabalho os aspectos da alimentação de Xenomelaniris brasiliensis (Quoy \& Gaimard, 1824), Tilapia rendalli (Boulenger, 1896), Jenynsia lineata (Jenyns, 1842), Poecilia vivipara (Bloch \& Schneider, 1801) e Phalloptychus januarius (Hensel, 1868), visando contribuir para o conhecimento das relações tróficas entre essas cinco espécies de peixes, consideradas as mais abundantes na Laguna de Jacarepaguá, segundo ANDREATA et al. (1992).

O estudo da alimentação de uma espécie de peixe no seu habitat, sugere subsídios para a criação dessa espécie em cativeiro ou a utilização da mesma como controladora de " $n$ " desequilibrios no meio ambiente. Segundo COSTA et al. (1987) tal estudo é considerado de fundamental importância para se conhecer toda uma estrutura trófica na qual os organismos se inserem. A dieta e a procura por alimento são fatores que regulam, ou pelo menos, influenciam a distribuição dos exemplares no habitat (SABINO \& CORRÊA E CASTRO 1990). De acordo com o alimento disponivel no ambiente o peixe pode mudar, tanto o seu comportamento quanto o seu padrão de migração (CARAgitsou \& PAPACONSTATINOU 1990). Segundo

1) Apoio CNPQ/FAPERJ (Bolsas de aperfeiçoamento e pré-mestrado, respectivamente).

2) Departamento de Biologia (NUPÉLIA), Fundação Universidade Estadual de Maringá. Campus Universitário, Av. Colombo 3690, 87020-900 Maringá, Paraná, Brasil.

3) Departamento de Biologia Animal. Universidade Santa Úrsula, Rua Fernando Ferrari 75, 22231-040 Rio de Janeiro, Rio de Janeiro, Brasil. 
LARKIN (1956), a competição caracteriza-se pela demanda de mais de um organismo pelos mesmos recursos do meio ambiente para o suprimento de suas necessidades imediatas, podendo ser causada por alimentação em comum, por espaço, ou por predação entre os competidores.

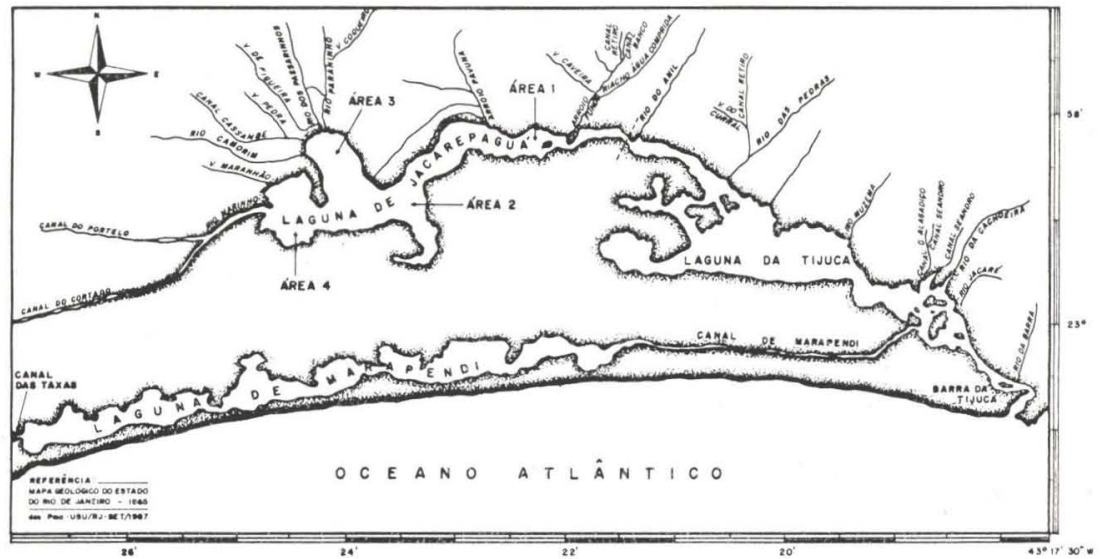

Fig. 1. Local da coleta, Laguna de Jacarepaguá.

A dieta alimentar, além de alguns aspectos da morfologia do tubo digestivo, de Xenomelaniris brasiliensis foi estudada por CARVALHO (1953). BEMVENUTI (1990) relacionou a dieta de duas espécies de peixe rei, durante as estações do ano. UNGER et al. (1984) estudaram a dieta de Xenomelaniris venezuelae (Eigenmann, 1920). Aspectos da alimentação dos poecilideos foram pesquisados por ZARET \& Rand (1970), Garcia Avila \& Gonzales Broche (1986), Reddy \& ShaKUNTALA (1979). SHIAU et al. (1989), experimentaram diferentes rações em duas espécies de Tilapia sp. VINYARD et al. (1988) estudaram o impacto de Tilapia galilae (Artedi, 1757) e Tilapia aurea (Steindachner, 1864) na comunidade plantônica. SANTIAGO et al. (1987) analisaram a influência da taxa alimentar e da dieta no crescimento e na sobrevivência de jovens de Oreochromis niloticus (Hasselquist, 1757). DiAnA et al. (1991) estudaram o efeito da tilápia do Nilo (Oreochromis niloticus) no ecossistema de tanques de aquacultura e sua significância na hipótese da cascata trófica.

\section{Área estudada}

A Laguna de Jacarepaguá localiza-se ao sul do Rio de Janeiro, entre a latitude $22^{\circ} 57^{\prime} \mathrm{S}$ e longitude $43^{\circ} 24^{\prime} \mathrm{W}$. Faz parte do Complexo Lagunar da Baixada de Jacarepaguá, e a sua única ligação com o Oceano Atlântico se faz pela laguna da Tijuca, através do Canal da Joatinga. Dentre os rios que desaguam na região, destacam-se Paraninho, Marinho, Camorim. A precária influência das águas oceânicas favorecem uma baixa salinidade, variando entre 0,0 e 8,0\%o e uma média de 2,0\%o (ANDREATA et al. 1992). 
A vegetação das margens é constituída por Typha dominguensis Pers, Acrosticum aureum Linnaeus, Cladium jamaicense Crantz, Laguncularia racemosa Gaertin., Aviscenia schaueriana Stapf. \& Leechman e Hibiscus tiliaceus Linnaeus, enquanto que a macrófita aquática flutuante dominante é Eichornia crassipes (Mart.) Solms (ARAÚJo 1978).

Segundo ANDREATA et al. (1992) esta laguna vem sofrendo um processo de descaracterização, devido a sucessivos aterros decorrentes da ocupação acelerada, além do aporte de esgotos e sedimento. STRANCH et al. (1982) afirmam que, o impacto causado por despejos urbanos e industriais in natura tem como principais contribuintes o Arroio Pavuna e o Canal do Riocentro. As conseqüências do lançamento desses efluentes nessa laguna são: a eutrofização (COELHO \& FONSECA 1981) e constantes mortandades de peixes (COUTINHO 1986; ANDREATA op cit.) e de outros organismos.

\section{MATERIAL E MÉTODOS}

As coletas foram mensais e diurnas, em quatro áreas da Laguna de Jacarepaguá (Fig. 1), durante o período de março de 1990 a fevereiro de 1991 . O instrumento utilizado para a captura foi o arrasto-de-praia com malhas $3 \mathrm{~mm}$ e $15 \mathrm{~mm}$.

No campo, os animais foram acondicionados em sacos plásticos e preservados em gelo para posterior análise. No laboratório os mesmos foram triados e fixados em formol a $10 \%$. Um total de 1015 exemplares foram eviscerados, sendo 204 de Xenomelaniris brasiliensis e separados em duas classes de comprimento padrão, entre 20 e $60 \mathrm{~mm}$ e entre 61 e $100 \mathrm{~mm}, 210$ espécimens de Jenynsia lineata entre 18 e $45 \mathrm{~mm}$ e entre 46 e $70 \mathrm{~mm}, 229$ de Tilapia rendalli entre 30 e $60 \mathrm{~mm}$ e entre 61 e $100 \mathrm{~mm}, 177$ de Poecilia vivipara entre 20 e $50 \mathrm{~mm}$ e 195 de Phalloptychus januarius entre 20 e $35 \mathrm{~mm}$. Após a evisceração dos especimens, obteve-se o peso úmido dos estômagos, sendo que para Xenomelaniris brasiliensis foi utilizado o trato digestivo (esôfago até o reto) por não possuir um estômago diferenciado.

Para a contagem dos itens microscópicos foi utilizada uma subamostra de cada estômago e analizada em câmara de contagem de células de Neubauer para uma estimativa numérica dos diferentes organismos contidos na placa de Petri. Os índices de freqüência (IF), numérico (IN) e de peso (IP) dos itens foi de acordo com Matallanas (1980), onde: $\mathrm{IF}=$ número de peixes que contém certa presa no estômago x 100/número total de peixes examinados, $\mathrm{IN}=$ percentagem entre o número de indivíduos de uma presa determinada e o número total dos indivíduos de todas as presas e IP = relação entre o peso total dos organismos de certa presa e o peso total de todas as presas, expresso em percentagem. O índice de importância relativa foi de acordo com PINKAS et al. (1971), onde IIR =IF x (IN + IP). Para a análise de agrupamento utilizou-se o coeficiente numérico dos itens ingeridos pelas cinco espécies de acordo com a classe de comprimento padrão, segundo a fórmula de MORISITA apud ZARET \& RAND (1970): 


$$
C \lambda=\frac{\sum_{i=1}^{s} X i Y i}{\sum_{i=1}^{s} X i^{2}+\sum_{i=1}^{s} Y i^{2}}
$$

onde: $\mathbf{S}=$ número total de categorias alimentares; $\mathbf{X i}=$ coeficiente numérico total para uma determinada categoria alimentar $\mathbf{i}$ para a espécie $\mathbf{X} ; \mathbf{Y i}=$ coeficiente numérico total para uma determinada categoria alimentar $\mathbf{i}$ para a espécie $\mathbf{Y}$. Segundo ZARET \& RAND (1970), qualquer valor maior ou igual a 0,60 possui uma sobreposição significativa.

Para as análises numéricas foram utilizados os programas Lotus 123 e NT-SYS.

Para evidenciar as estruturas das algas foram utilizados a solução aquosa de azul de metileno a $4 \%$, para detectar a presença de bainha mucilaginosa e lugol para observação de pirenóides, segundo a metodologia adotada por CHAMIXAES (1990). A identificação a nível genérico das algas e dos demais organismos foi baseada principalmente em Newell \& NewEll (1963), GarnetT (1965), Borror \& Delong (1969), Bourelly (1972), MACAN (1975), NEEdHAM \& NEEDHAM (1978).

\section{RESULTADOS}

\section{ESPECTRO ALIMENTAR}

Xenomelaniris brasiliensis foi considerada omnívora por possuir uma dieta variada, sendo que os maiores índices de freqüência e numérico para aqueles medindo entre 20 e $60 \mathrm{~mm}$ foram representados pelos copépodos do tipo ciclopóides e "detrito-areia" (grãos de areia, matéria orgânica em decomposição, carapaças); para aqueles entre 61 e $100 \mathrm{~mm}$, os insetos alcançaram os maiores índices, principalmente himenópteros, seguidos dos dípteros (Tab. I). Os insetos também apresentaram o maior índice de importância relativa para as duas classes de comprimento (Tab. I). Os himenópteros foram representados pelos formicídeos alóctones, os dípteros pelos nematóceros autóctones e os coleópteros pelos hidrofilídeos.

Jenynsia lineara se revelou omnívora por possuir uma dieta variada. Para os exemplares medindo entre 18 e $45 \mathrm{~mm}$ os itens copépodo e "detrito-areia" (grãos de areia e matéria orgânica em decomposição) apresentaram os maiores índices de freqüência; enquanto que as larvas de insetos e "detrito-areia" apresentaram os maiores índices de importância relativa. Para aqueles entre 46 e $70 \mathrm{~mm}$, os insetos dípteros adultos e "detrito-areia" foram mais freqüentes, abundantes e de maior importância relativa (Tab. II).

Tilapia rendalli foi considerada fitófaga por se alimentar preferencialmente de algas, que foram os itens mais freqüentes, abundantes e de maior importância relativa para os dois intervalos de comprimento (Tab. III). Dentre as cianofíceas, 
Tab. I. Índices de freqüência (IF), de peso (IP), numérico (IN) e de importância relativa (IIR) dos itens alimentares de Xenomelaniris brasiliensis com comprimento padrão (CP) entre 20 e $60 \mathrm{~mm}$ e entre 61 e $100 \mathrm{~mm}$.

\begin{tabular}{|c|c|c|c|c|c|c|c|c|}
\hline \multirow{2}{*}{ Classe de CP } & \multicolumn{4}{|c|}{$20-60 \mathrm{~mm}$} & \multicolumn{4}{|c|}{$61 \vdash 100 \mathrm{~mm}$} \\
\hline & IF & IP & IN & IIR & IF & IP & IN & IIR \\
\hline ALGAS & 36,60 & $\cdot$ & 19.20 & 702,70 & 24,6 & . & 1,80 & 44,3 \\
\hline Cyanophyta & - & - & 6,44 & - & - & - & 0,65 & - \\
\hline Chrysophyta & & - & 6,64 & - & - & - & 0,63 & - \\
\hline Chlorophyta & - & - & 6,11 & - & - & . & 0,49 & - \\
\hline PEIXES & 5,63 & 1,48 & 0,28 & 9,90 & 34,5 & 3,87 & 3,31 & 247,7 \\
\hline COPEPODOS & 70,42 & - & 30,14 & 2122,40 & 33,3 & - & 9,05 & 301,3 \\
\hline POLIQUETOS & 19,71 & 3,12 & 0.50 & 71,36 & 37.0 & 5,66 & 3,33 & 332,2 \\
\hline INSETOS ADULTOS & 46,47 & 21,38 & 3,84 & 1171,90 & 82,7 & 72,86 & 42,40 & 9531,1 \\
\hline Diptera & - & - & 3,84 & - & - & - & 14,46 & - \\
\hline Hymenoptera & . & - & - & - & - & - & 25,17 & - \\
\hline Coleoptera & - & - & . & . & - & " & 2,70 & - \\
\hline LARVAS DE INSETOS & 54,90 & 40,28 & 7,18 & 2605,50 & 37,0 & 7,83 & 4,62 & 460,6 \\
\hline MOLUSCOS & 38,00 & 13,16 & 5,31 & 701,40 & 25,9 & 5,12 & 7,30 & 321,6 \\
\hline DETRITOS DE AREIA & 90,10 & 20,55 & 29,22 & 4484,20 & 81,4 & 4,37 & 25,07 & 2396,4 \\
\hline SEMENTES & & . & . & - & 3,7 & 0,27 & 0,70 & 3,6 \\
\hline OUTROS & 22,50 & - & 4,33 & 97,40 & 16,0 & - & 2,44 & 39,0 \\
\hline $\mathrm{N}^{\circ}$ de tratos examinados & & & & & & & & \\
\hline $\mathrm{N}^{\circ}$ de tratos vazios & & & & & & & & \\
\hline
\end{tabular}

consideradas as mais numerosas, destacaram-se Mycrocistis sp., seguida de Lyngbia sp. e Oscillatoria sp. As crisofíceas foram representadas pelas diatomáceas cêntricas, especialmente Thalassiosira sp. e Chaetoceros sp., e pelas penadas como Nitzchia sp., Frustulia sp. e Gomphonema sp. As clorofíceas mais representativas foram Volvox sp., Scenedesmus sp. e Zigogonium sp. Dentre os insetos, os coleópteros hidrofilídeos foram observados em apenas cinco espécimes do total analisado. O item "outros" foi composto principalmente por fragmentos de folhas de angiospermas e zoósporos.

Poecilia vivipara foi considerada iliófaga, sendo que as algas perifíticas epibênticas e "detrito-areia" foram os alimentos mais frequentes, abundantes e de maior importância relativa para a classe de comprimento entre 20 e $50 \mathrm{~mm}$ (Tab. IV). Dentre as cianofíceas, Microcystis sp. se destacaram numericamente.

Phalloptychus januarius também foi considerada iliófaga com a dieta composta principalmente de algas cianofíceas e clorofíceas perifíticas. As algas epibênticas filamentosas como Lyngbia sp. e Oscillatoria sp. e coloniais como Mycrocistis sp. e Volvox sp. e "detrito-areia" alcançaram os maiores índices de freqüência, numérico e de importância relativa para a classe de comprimento entre 20 e $35 \mathrm{~mm}$ (Tab. V). 
Tab. II. Índices de freqüência (IF), de peso (I P), numérico (IN) e de importância relativa (IIR) dos itens alimentares de Jenynsia lineata com comprimento padrão (CP) entre 18 e $45 \mathrm{~mm}$ e entre $46 \mathrm{e}$ $70 \mathrm{~mm}$.

\begin{tabular}{|c|c|c|c|c|c|c|c|c|}
\hline \multirow{2}{*}{ Classe de CP } & \multicolumn{4}{|c|}{$18 \longmapsto 46 \mathrm{~mm}$} & \multicolumn{4}{|c|}{$46-70 \mathrm{~mm}$} \\
\hline & IF & IP & IN & IIR & IF & IP & IN & IIR \\
\hline ALGAS & 38,5 & - & 21,680 & 830,8 & 24,2 & $\cdot$ & 14,400 & 348,5 \\
\hline Cyanophyta & • & - & 13,900 & - & • & . & 11,430 & $\cdot$ \\
\hline Chrysophyta & - & - & 6,970 & - & - & - & 2,500 & - \\
\hline Chlorophyta & - & - & 0,710 & . & . & - & 0,470 & \\
\hline CRUSTÁCEOS & 65,7 & - & 21,460 & 1405,9 & 54,2 & - & 15,300 & 829,2 \\
\hline Cladóceros & - & - & 0,005 & - & - & - & $\cdot$ & $\cdot$ \\
\hline Copépodos & - & . & 21,450 & - & - & - & 15,300 & . \\
\hline Decápodos & - & - & 0,012 & - & - & - & $\cdot$ & - \\
\hline VERMES & 41,4 & 10,3 & 2,300 & 521,8 & 42,8 & 5,1 & 1,630 & 288,9 \\
\hline Poliquetos & - & · & 2,170 & - & - & - & 1,530 & - \\
\hline Rotiferos & - & $\cdot$ & 0,137 & - & - & - & 0,100 & \\
\hline INSETOS ADULTOS & 45,7 & 30,9 & 7,390 & 1749,8 & 77,1 & 48,7 & 16,120 & 4997,6 \\
\hline Diptera & - & $\cdot$ & 5,370 & - & - & - & 15,900 & - \\
\hline Hymenoptera & - & - & 0.025 & . & . & - & 0,220 & - \\
\hline Coleoptera & - & - & $\cdot$ & - & - & . & 0,006 & - \\
\hline PUPAS DE INSETOS & 62,8 & 22,6 & 5,570 & 1487.3 & 48,5 & 20,5 & 6,660 & 1317,7 \\
\hline LARVAS DE INSETOS & 62,8 & 27,7 & 6,030 & 1992,6 & 65,7 & 16,1 & 6,100 & 1461,8 \\
\hline MOLUSCOS & 32,8 & 4,1 & 6,020 & 332.6 & 37,1 & 4,1 & 10,890 & 556,1 \\
\hline DETRITOS DE AREIA & 87,1 & 6,2 & 27,900 & 2968,3 & 71,4 & 7.7 & 27,500 & 2475,4 \\
\hline OUTROS & 12,8 & & 1,700 & 21,7 & 14,2 & - & 1,130 & 16,0 \\
\hline$N^{0}$ de estômagos examinados & & & & & & & & \\
\hline$N^{0}$ de estômagos vazios & & & & & & & & \\
\hline
\end{tabular}

\section{ANÁLISE DE AGRUPAMENTOS}

A análise de agrupamentos mostrou um ponto de separação na dieta das cinco espécies, com a formação de dois grupos principais (Fig. 2), unidos pela similaridade numérica de itens alimentares. O grupo 1 formado por Xenomelaniris brasiliensis e Jenynsia lineata, omnívoros, e o grupo 2 agrupando Poecilia vivipara, Phalloptychus jamuarius e Tilapia rendalli, que se alimentaram preferencialmente de microalgas perifíticas e "detrito-areia" (iliófagos). Xenomelaniris brasiliensis e $J$. lineata entre 61 e $100 \mathrm{~mm}$ e entre 46 e $70 \mathrm{~mm}$ respectivamente, preferiram os insetos adultos, apresentando uma sobreposição de $99 \%$, enquanto que $J$. lineata entre 18 e $45 \mathrm{~mm}$, as larvas. Xenomelaniris brasiliensis entre $20 \mathrm{e}$ $60 \mathrm{~mm}$, apresentou o maior número de copépodos em seu trato digestivo e deste modo, a sobreposição foi de $76 \%$. Para $T$. rendalli não houve diferença de hábitos alimentares entre as duas classes de comprimento estudadas, se alimentando basicamente do lodo. 


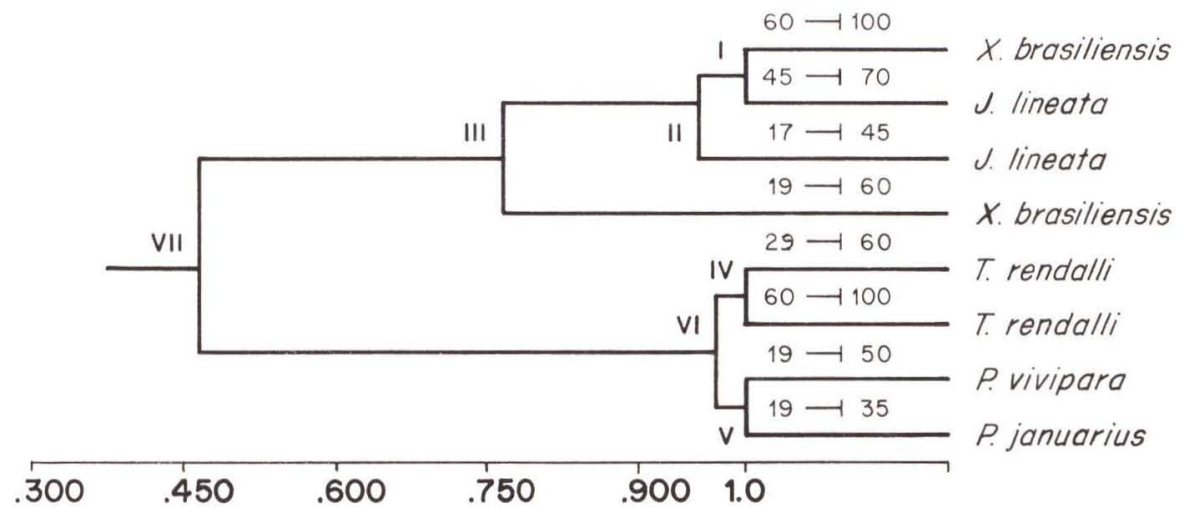

Fig. 2. Agrupamento das espécies de peixes estudadas, por classe de comprimento padrão, segundo MORISITA apud ZARET \& RAND (1970).

Tab. III. Índices de freqüência (IF), de peso (IP), numérico (IN) e de importância relativa (IIR) dos itens alimentares de Tilapia rendalli com comprimento padrão (CP) entre 30 e $60 \mathrm{~mm}$ e entre 61 e $100 \mathrm{~mm}$.

\begin{tabular}{|c|c|c|c|c|c|c|c|c|}
\hline \multirow{2}{*}{ Classe de CP } & \multicolumn{4}{|c|}{$30 \vdash 60 \mathrm{~mm}$} & \multicolumn{4}{|c|}{$61 \longmapsto 100 \mathrm{~mm}$} \\
\hline & IF & IP & IN & $\| R$ & IF & IP & IN & $I ! R$ \\
\hline ALGAS & 100,00 & - & 76,68 & 7665,0 & 100.0 & - & 70,86 & 7086,00 \\
\hline Cyanophyta & - & & 54,67 & & - & - & 54,67 & - \\
\hline Chrysophyta & - & - & 13,96 & - & - & - & 13,96 & - \\
\hline Chlorophyta & - & - & 8,06 & - & - & - & 8,05 & - \\
\hline COPÉPODOS & 63,60 & - & 11,21 & 712,9 & 64,8 & - & 13,87 & 898,70 \\
\hline ROTIFEROS & 36,36 & - & 1,86 & 67,6 & 36,0 & - & 3,63 & 130,60 \\
\hline DETRITOS DE AREIA & 46,46 & 71,42 & 5,55 & 3490,2 & 45,0 & 89,28 & 7.23 & 4342,90 \\
\hline MOLUSCOS & 7,27 & 28,57 & 1,40 & 217,8 & 37,8 & 6,00 & 1,81 & 257,40 \\
\hline COLEÓPTEROS & & & & & 4,5 & 5,71 & 0,03 & 25,80 \\
\hline OUTROS & 36,36 & - & 3,22 & 117,0 & 36,0 & - & 1,98 & 71,28 \\
\hline $\mathrm{N}^{\circ}$ de estômagos examinados & \multicolumn{4}{|c|}{112} & \multicolumn{4}{|c|}{113} \\
\hline $\mathrm{N}^{\circ}$ de estômagos vazios & \multicolumn{4}{|c|}{2} & \multicolumn{4}{|c|}{2} \\
\hline
\end{tabular}

\section{DISCUSSÃO}

Apesar da sobreposição alimentar expressiva entre as espécies examinadas, essas convivem na laguna de Jacarepaguá pois possuem diferentes nichos alimentares, além de o recurso alimentar não ser um fator limitante para as mesmas.

Os despejos de efluentes domésticos e industriais in natura na Laguna, que não podem ser totalmente decompostos e dissipados pelo meio, favorecem a proliferação das algas. Dentre esssas, as cianofíceas, representadas principalmente 
Tab. IV. Índices de freqüência (IF), de peso (IP), numérico (IN), e de importância relativa (IIR) dos itens alimentares de Poecilia vivipara com comprimento padrão entre 20 e $50 \mathrm{~mm}$.

\begin{tabular}{|c|c|c|c|c|}
\hline \multirow{2}{*}{ Classe de CP } & \multicolumn{4}{|c|}{$20 \mapsto 50 \mathrm{~mm}$} \\
\hline & IF & IP & IN & IIR \\
\hline ALGAS & 42,7 & - & 63,870 & 2454,8 \\
\hline Cyanophyta & - & - & 44,700 & - \\
\hline Chrysophyta & - & - & 13,450 & - \\
\hline Chlorophyta & - & - & 5,720 & - \\
\hline COPÉPODOS & 51,3 & - & 9,290 & 476,5 \\
\hline INSETOS ADULTOS & 12,8 & 8,27 & 0,520 & 112,5 \\
\hline Diptera & - & - & 0,102 & - \\
\hline Hymenoptera & - & - & 0,415 & - \\
\hline LARVAS DE INSETOS & 14,5 & 17,21 & 1,040 & 264,6 \\
\hline ROTÍFEROS & 9,4 & - & 0,500 & 4,7 \\
\hline DETRITOS DE AREIA & 87,1 & 74,50 & 27,030 & 8843,2 \\
\hline OUTROS & 19,6 & - & 3,720 & 72,9 \\
\hline$N^{\circ}$ de estômagos examinados & & & & \\
\hline$N^{\circ}$ de estômagos vazios & & & & \\
\hline
\end{tabular}

Tab. V. indices de freqüência (IF), de peso (IP), numérico (IN) e de importância relativa (IIR) dos itens alimentares de Phalloptychus januarius com comprimento padrão (CP) entre 20 e $35 \mathrm{~mm}$.

\begin{tabular}{|c|c|c|c|c|}
\hline \multirow{2}{*}{ Classe de CP } & \multicolumn{4}{|c|}{$20 \vdash 35 \mathrm{~mm}$} \\
\hline & IF & IP & IN & $\| R$ \\
\hline ALGAS & 96,90 & - & 65,97 & 6392,7 \\
\hline Cyanophyta & - & - & 36,78 & - \\
\hline Chrysophyta & - & - & 19,22 & - \\
\hline Chlorophyta & - & & 9,97 & - \\
\hline COPÉPODOS & 42,26 & - & 12,44 & 525,7 \\
\hline DETRITOS DE AREIA & 76,16 & 68,62 & 18,09 & 6256,9 \\
\hline ROTÍFEROS & 22,60 & - & 1,90 & 42,9 \\
\hline LARVAS DE INSETOS & 15,46 & 31,37 & 0,04 & 485,5 \\
\hline OUTROS & 22,60 & - & 1,52 & 34,35 \\
\hline $\mathrm{N}^{\circ}$ de estômagos examinados & & & & \\
\hline $\mathrm{N}^{\circ}$ de estômagos vazios & & & & \\
\hline
\end{tabular}

Revta bras. Zool. 11 (4): $789-800,1994$ 
por Mycrocistis sp., são as dominantes na alimentação de Tilapia rendalli, Poecilia vivipara e Phalloptychus januarius. Os despejos de detritos orgânicos também favorecem a proliferação de insetos, principalmente nematóceros, que apesar de serem alimento para Xenomelaniris brasiliensis e Jenynsia lineata são também vetores de doenças, além de causarem incômodos a população local (NEHAB \& BARBOSA 1984.).

Estudos da toxidez da alga Microcystis aeruginosa foram realizados por alguns autores como CODD et al. (1989) e FALCONER (1989), entre outros. Segundo ROBARTS (1984), a maioria das colônias de Microcystis sp. é muito grande para ser ingerida pelo zooplâncton. O que se observou na laguna em questão é que os peixes filtradores e zooplanctônicos não são dominantes, corroborando o que foi verificado por ROBARTS ( $o p$ cit.) na represa de Hartbeespoort, onde ocorre a dominância de Microcystis aeruginosa. As cinco espécies estudadas se alimentaram basicamente de detritos orgânicos, algas perifíticas e invertebrados bentônicos, com excessão de Xenomelaniris brasiliensis e Jenynsia lineata que, a partir de 75 e $55 \mathrm{~mm}$ respectivamente, alimentaram-se basicamente de insetos adultos.

ANDREATA et al. (1992), que fizeram um estudo da abundância e diversidade da ictiofauna da Laguna de Jacarepaguá, utilizando quatro instrumentos de coleta, verificaram que das 15 espécies encontradas, as cinco estudadas nesse trabalho representaram 90\% do total de captura. ANDREATA et al. (1992) verificaram, portanto, uma diversidade baixa, com 1,708.

Os dados apontados no trabalho indicam que, provavelmente, o fluxo de energia no sistema tenha uma base detrital, o que é observado nos índices de importância relativa e na sobreposição significativa das espécies pelos detritos orgânicos. Embora sejam necessários novos estudos, incluindo aqueles sobre a importância da $T$. rendalli como uma provável controladora da proliferação de algas, a despeito dos estudos que já foram realizados por VINYARD et al. (1987) no lago Kinneret em Israel. VINYARD et al. (1987) observaram que Tilapia galilae poderia reduzir a abundância algal, contribuindo para a melhoria da qualidade da água.

AGRADECIMENTOS. Agradecemos as professoras Dra. Isabel de Fátima Andrian, da Universidade Estadual de Maringá e Dra. Érica Pellegrini Caramaschi, da Universidade Federal do Rio de Janeiro pelas crílicas e sugestẽes ao manuscrito; ao professor Mauro José Cavalcanti, da Universidade Santa Úrsula, pela revisão dos dados estatísticos; aos biólogos Cláudio Lopes Soares e Alex Garcia Marca, pelo auxílio nas coletas, e a professora Dra. Joanete Maron Ramos, chanceler e chefe de pesquisa da Universidade Santa Úrsula, pelo apoio e incentivo às nossas pesquisas.

\section{REFERÊNCIAS BIBLIOGRÁFICAS}

Andreata, J.V.; A.M. Saad; L.A. De Moraes; C.L. Soares \& A.G. Marca. 1992. Associação, similaridades e abundância relativa dos peixes da Laguna de Jacarepaguá, Rio de Janeiro, Brasil. Bol. Mus. Nac., Rio de Janeiro, 335: $1-25$.

ARAÚJO, D.S. DE. 1978. As comunidades vegetais das margens das Lagoas de 
Jacarepaguá, Rio de Janeiro. Rio de Janeiro, Fundação Estadual de Engenharia do Meio Ambiente (FEEMA), série técnica 3, 35p.

Bemvenuti, M. DE A. 1990. Hábitos alimentares de peixes-rei (Atherinidae) na região estuarina da Lagoa dos Patos, RS, Brasil. Atlântica, Rio Grande, 12 (1): 79-102.

Bloch, M.E. \& G. Schneider. 1801. Systema ichthyologiae iconibus ex illustratum. Berolini, 584p.

Borror, D.J. \& D.M. Delong. 1969. Estudo dos Insetos. São Paulo, Editora da Universidade de São Paulo, 653p.

Boulenger, G.A. 1896. Description of new fishes from the upper Shiré River, British Central Africa, collected by Dr. Percy Kendall, and presented to the British Museum by Sir Hany H. Johnson, K.C.B.. Proc. Zool. Soc. London 4: 915-920.

Bourelly, P. 1972. Les algues d'eu douce - iniciation à la systematique. Paris, Ed. N. Bubéc Science, 1531 p.

Caragitsou, E. \& C. Papaconstatinou. 1990. Food and feeding habits of large scale gurnard, Lepidotrigla cavillone (Triglidae) in Greek seas. Cybium, Paris, 14 (2): 95-104.

CARVAlHO, J.P. 1953. Alimentação de Xenomelanmiris brasiliensis (Quoy \& Gaimard) pisces Mugiloidei - Atherinidae. Bol. Inst. Oceanogr. Universidade de São Paulo, 4 (2): 126-144.

Chamixaes, C.B.C.B. 1990. Ficotlórula do açude de Apipucos (Recife-PE), Rev. Bras. Biol. 50 (1): 45-60.

Codd, G.A., S.G. Bell \& W.P. Brooks. 1989. Cyanobacterial toxins in water. Water Sci. Technol., London, 21: 1-13.

Coelho, V.M.B. \& M.R.M.B. FonseCA. 1981. Problemas da eutrofização no Estado do Rio de Janeiro. Rio de Janeiro, Fundação Estadual de Engenharia do Meio Ambiente, série congressos, 51p.

Costa, T.L.M.; A. DE L. Vasconcelos Filho \& E.M.B. Galizaviana. 1987. Aspectos sobre a alimentação do Cângulo, Balistes vetula Linnaeus 1959 (Pisces Balistidae) no Estado de Pernambuco, Brasil. Rev. bras. Zool. 4 (2): 71-88.

Coutinho, P.N.C. 1986. Sugestões para o gerenciamento de estuários. Arq. Ciênc. Mar, Fortaleza, 25: 77-86.

Diana, J.S., D.J. DetTweiler \& C.K. Lin. 1991. Effect of Nile tilapia (Oreochromis niloticus) on the ecosystem of aquaculture ponds, and its significance to the trophic cascade hypothesis. Can. J. Fish Aquat. Sci. 48: 183-191.

FALCONER, I.R. 1989. Effects on human health of some toxic cyanobacteria (blue green algae) in reservoirs. Toxic Asses. Int., New York, 4: 175-184.

Garcia Avila, I. \& R. Gonçales Broche. 1986. Principales especies de peces larvivoros en la familia Poecillidae y su efectividad en les condiciones naturales de Cuba. Rev. Cubana Med. Trop. 38 (2): 197-202.

GaRnetT, W.J. 1965. Freshwater Microscopy, London, Constable \& Company, 
$375 p$.

HENSEL, R.F. 1968. Beitraege zur Kenntniss der Wirbeltiere Suedbrasiliens. Fische. Archiv. Naturg. 34 (1): 356-375.

JENYNS, L. 1842. Fish. In: C. DARWIN (ed.). The zoology of the voyages of the H.M.S. Beagle, 1839, 43. London, 4: 1-172.

LARKIN, P.A. 1956. Interespecific competition and population control in freshwater fish. J. Fish Res. Bd., Ottawa, 13 (3): 327-342.

MACAN, T.T. 1975. Invertebrates de agua dulce. Madrid, Editiones Universidad de Navana, $118 \mathrm{p}$.

Matallanas, J. 1980. Étude de lalimentation dOphidion barbatum (Pisces, Ophidiidae) dans la mer Catalane. Cybium, Paris, 10: 81-89.

Needham, J.G. \& P.R. Needham. 1978. Los seres vivos de las águas dulces. Spain, Editorial Reverté, $131 \mathrm{p}$.

Nehab, M.A.F. \& L.R. Barbosa. 1984. Quadro da situação da Baixada de Jacarepaguá, Rio de Janeiro. Rio de Janeiro, Fundação Estadual de Engenharia do Meio Ambiente, 107p.

Newell, G.E. \& R.C. Newell. 1963. Marine plankton: a practical guide. London, Anchor Press, 244p.

PinKas, L; M.S. Oliphant \& L.K.IVERSON. 1971. Food habits of albacore, bluefin tuna, and bonito in California waters. Calif. Dep. Fish. Game Fish Bull. 152: 105.

QuOY, J.R.C. \& P. GaIMARD. 1824. Zoologie. In: L.C.D. DE FreyCinet (ed.). Voyage autour du monde... executé sur... l'Úranie et la physicienne, pendant... 1817-1820., Paris, 1-2: 192-401.

REDdy, S.R. \& K. Shakuntala. 1979. Comparative studies on the food intake, growth and food convertion of two larvivorous fishes. Proc. Indian Acad. Sci. 88 (1): 425- 432.

RoBarTS, R.D. 1984. Hipertrophy, a consequence of development. Intern. J. Environmental Studies 25: 167-175.

SABINO, J. \& R.M. CORRÊA E CASTRO. 1990. Alimentação, período de atividade e distribuição espacial dos peixes de um riacho da Floresta Atlântica (sudeste do Brasil). Rev. Bras. Biol. 50 (1): 23-36.

Santiago, C.B.; M.B. AldabA \& O.S. Reyes. 1987. Influence of feeding rate and diet form on growth and survival of Nile tilapia (Oreochromis niloticus) fry. Aquaculture, Amsterdã, 64: 277-282.

Shiau, S.Y.; C.C. KwoK; J.Y. Hwang; C.M. Chen \& S.L. Lee. 1989. Replacement of fishmeal with soybean meal in male Tilapia (Oreochromis niloticus x $O$. (aureus) fingerling diets at a subotimal protein level. J. World Aquac. Soc. 20 (4): 230-235.

Stranch, C.E.; C.C. Garcia; G.F. Sampaio; L. DE A. Chacesado; M. Funfe; P.R.P. Araujo; S. Utchitel; V.A. Lage \& Z. DE O. SoARes. 1982. Estudo do Complexo Lagunar da Barra da Tijuca (Fase I: Lagoa de Jacarepaguá), Rio de Janeiro. Rio de Janeiro, Fundação Estadual de Engenharia do Meio Ambiente, 37p. 
Unger, P.A.; W.M. LeWIS JR. \& D.H. MCCLEAN 1984. Non visual feeding in a visual planktivore, Xenomelaniris venezuelae. Oecologia 64 (2): 280-283.

Vinyard, G.L.; R.W. Drenner; M. Gophen; U. Pollingher; D.L. WinkelMAN; K.D. HAMBRIGHT. 1988. An experimental study of plancton community impacts of two omnivorous filter-feeding cichlids, Tilapia galilaea and Tilapia aurea. Can. J. Aquat. Sci. 45: 685-690.

ZARET, T.M. \& S. RAND. 1970. Competition in tropical stream fishes: support for competitive exclusion principle. Ecology 52: 336-342.

Recebido em 30.IV.1994; aceito em 01.XII.1994. 